\title{
Bandgap-assisted quantum control of topological edge states in a cavity
}

\author{
Wei Nie ${ }^{1,2}$ and Yu-xi Liu ${ }^{1,2, *}$ \\ ${ }^{1}$ Institute of Microelectronics, Tsinghua University, Beijing 100084, China \\ ${ }^{2}$ Frontier Science Center for Quantum Information, Beijing 100084, China
}

(Received 5 July 2019; accepted 2 March 2020; published 24 March 2020)

\begin{abstract}
Quantum matter with exotic topological order has potential applications in quantum computation. However, in present experiments, the manipulations on topological states are still challenging. We here propose an architecture for optical control of topological matter. We consider a topological superconducting qubit array with a Su-Schrieffer-Heeger (SSH) Hamiltonian which couples to a microwave cavity. Based on parity properties of the topological qubit array, we propose an optical spectroscopy method to observe the topological phase transition, i.e., edge-to-bulk transition. This new method can be achieved by designing cavity-qubit couplings. A main purpose of this work is to understand how the topological phase transition affects light-matter interaction. We find that the topological bandgap plays an essential role on this issue. In the topological phase, the resonant vacuum Rabi splitting of degenerate edge states coupling to the cavity field is protected from those of bulk states by the bandgap. In the dispersive regime, the cavity induced coupling between edge states is dominant over couplings between edge and bulk states, due to the topological bandgap. As a result, quantum interference between topological edge states occurs and enables single-photon transport through boundaries of the topological qubit array. Our work may pave a way for topological quantum state engineering.
\end{abstract}

DOI: 10.1103/PhysRevResearch.2.012076

Introduction. Characterization of topological matter is a crucial issue in condensed matter physics [1]. A hallmark of topological phases is the existence of topological invariants, e.g., Chern number and Zak phase, defined on energy bands of the systems [2-4]. According to edge-bulk correspondence, topological states emerge in the bandgaps and give rise to many novel transport phenomena $[5,6]$. Due to their insensitivity to local decoherence, topological states have prospective applications in quantum information processing. In particular, zero-dimensional edge states, e.g., Majorana bound states, are candidates to realize topological quantum computation [7-9] and have been observed experimentally in a range of materials, including semiconductor nanowires [10-13], ferromagnetic atomic chains [14], and iron-based superconductors [15]. However, the manipulations of edge states are rather challenging, for which reason topological materials with large bandgaps are explored [16-18].

Cavity quantum electrodynamics (QED), in which quantized electromagnetic fields are strongly coupled to an atomic system, was originally used for studying fundamentals of atomic physics and quantum optics [19]. With superb control of quantum states, cavity QED is now applied to quantum information processing, in which the cavity field is proposed

\footnotetext{
*yuxiliu@mail.tsinghua.edu.cn

Published by the American Physical Society under the terms of the Creative Commons Attribution 4.0 International license. Further distribution of this work must maintain attribution to the author(s) and the published article's title, journal citation, and DOI.
}

for manipulating, measuring, or transferring quantum states of atomic systems [20,21]. Circuit QED, in which a microwave transmission line resonator acting as a cavity is coupled to superconducting quantum circuit, is an extension of cavity QED [22,23]. The on-chip circuit QED system is not only a good platform for studying fundamental physics in the microwave regime [24], but also a very promising candidate for realizing quantum computation and simulations [25-42]. In particular, one-dimensional (1D) qubit arrays have been used to explore many-body localization [38,39], Mott insulators of photons [41], and correlated quantum walks [42]. Moreover, superconducting qubit systems also offer hope to simulate topological matter [43-47].

In this work, we study the interaction between a microwave cavity and a topological superconducting qubit array, described by the Su-Schrieffer-Heeger (SSH) model [48], which has been experimentally realized [49-51]. Differently from the electronic transport detections of Majorana fermions [13,52-54], the cavity spectroscopy method we study here unveils the edge states and topological phase transition with proper cavity-qubit couplings. We pinpoint the role of topological bandgap in quantum manipulation of edge states, especially for small qubit arrays.

Spectroscopic characterization of a topological qubit array by a cavity. As schematically shown in Fig. 1(a), we study that a 1D topological qubit array [55], with SSH interactions, is placed inside a cavity. Considering rapid progress and flexible chip designs of superconducting quantum circuits, we here assume that the $\mathrm{SSH}$ array with $N$ unit cells, formed by $2 N$ superconducting qubits [44], e.g., Xmon qubits [35,38], is coupled to a microwave transmission line resonator, as shown 


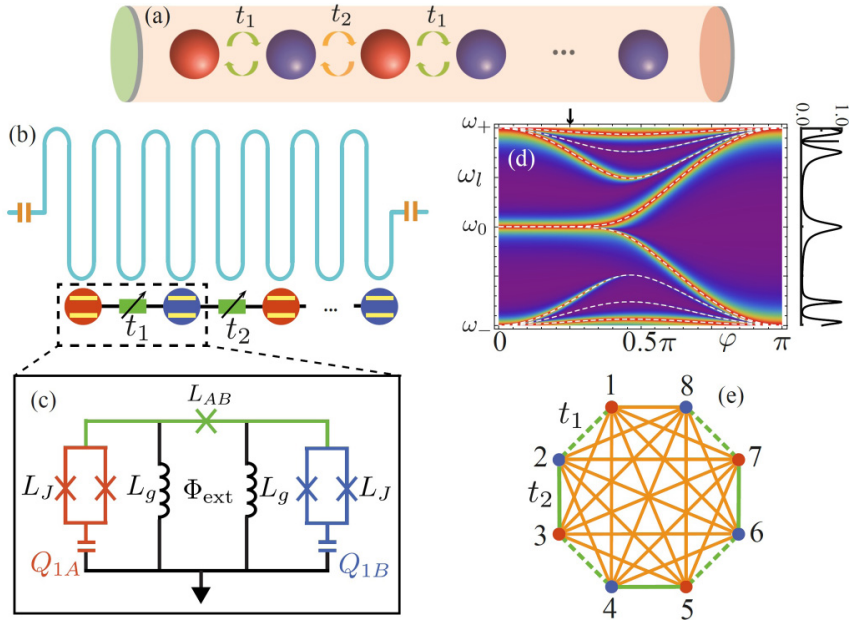

FIG. 1. (a) Schematic diagram for the SSH qubit array with dimerized couplings $t_{1}$ and $t_{2}$ placed inside a cavity. Red and blue balls represent $A$ and $B$ qubits, respectively. (b) Design of (a) with superconducting qubit circuits where the couplings $t_{1}$ and $t_{2}$ are tunable. The microwave transmission line resonator acting as a cavity is coupled to the qubits. (c) The first unit cell with tunable qubit-qubit coupling via a Josephson junction. $\Phi_{\text {ext }}$ is an external magnetic flux bias that controls the coupling (see Supplemental Material [64]). (d) Reflection spectrum of the qubit array with eight qubits. The frequencies of qubits and driving field are respectively $\omega_{0}$ and $\omega_{l} ; \omega_{ \pm}=\omega_{0} \pm 2 t_{0}$. The reflection at $\varphi=0.25 \pi$ is shown in the right panel. Here we consider cavity-qubit couplings $\boldsymbol{g}=$ $g_{0}(-1,1,1,1,-1,1,1,1)$ with $g_{0} / 2 \pi=5 \mathrm{MHz}$. Other parameters are $\omega_{0} / 2 \pi=6 \mathrm{GHz}, t_{0} / 2 \pi=100 \mathrm{MHz}, \kappa / 2 \pi=10 \mathrm{MHz}, \gamma_{i A}=$ $\gamma_{i B}=20 \times 2 \pi \mathrm{kHz}$. The white-dashed curves represent the energy spectrum of the qubit array. (e) Cavity mediated couplings between qubits, denoted by the orange lines, in the dispersive regime.

in Fig. 1(b). The Hamiltonian of the whole system is

$$
\begin{aligned}
H / \hbar= & \omega_{c} \hat{a}^{\dagger} \hat{a}+\sum_{i=1, \mu=A, B}^{i=N}\left(\omega_{0} \sigma_{i \mu}^{+} \sigma_{i \mu}^{-}+g_{i \mu} \sigma_{i \mu}^{+} \hat{a}+g_{i \mu}^{*} \hat{a}^{\dagger} \sigma_{i \mu}^{-}\right) \\
& +\sum_{i=1}^{i=N}\left(t_{1} \sigma_{i A}^{+} \sigma_{i B}^{-}+t_{2} \sigma_{i+1 A}^{+} \sigma_{i B}^{-}+\text {H.c. }\right)
\end{aligned}
$$

where $\omega_{c}$ and $\omega_{0}$ are the frequencies of the cavity and qubits, respectively. The parameter $g_{i \mu}$ denotes the coupling strength of the cavity to the qubit $\mu$ in the $i$ th unit cell. The operators of qubits $A$ and $B$ at the $i$ th unit cell are $\sigma_{i A}^{+}=\left|A_{i}\right\rangle\left\langle\alpha_{i}\right|$ and $\sigma_{i B}^{+}=$ $\left|B_{i}\right\rangle\left\langle\beta_{i}\right|$ with the ground (excited) states $\left|\alpha_{i}\right\rangle\left(\left|A_{i}\right\rangle\right)$ and $\left|\beta_{i}\right\rangle$ $\left(\left|B_{i}\right\rangle\right)$, respectively. The second line in Eq. (1) represents the SSH interaction Hamiltonian with tunable coupling strengths $t_{1}$ and $t_{2}$, which could be implemented in different ways in superconducting qubit circuits [38,39,56-63]. We here assume that controllable coupling between qubits is realized via a Josephson junction, which is biased by an external magnetic flux [62,63], as shown in Fig. 1(c). The coupling strengths are $t_{1}=t_{0}(1-\cos \varphi)$ and $t_{2}=t_{0}(1+\cos \varphi)$ with a tunable parameter $\varphi$ [64]. Note that the topological phase transition takes place at $\varphi=\pi / 2\left(t_{1}=t_{2}\right)$. The cases for $t_{1}<t_{2}$ and $t_{1}>t_{2}$ correspond to topological and nontopological phases, respectively.
To measure the topological qubit array, we assume that a weak probe field with the strength $\eta$ and the frequency $\omega_{l}=\omega_{c}$ is applied to the qubit array via the cavity. Thus, the dynamics of the reduced density matrix $\rho$ of the whole system can be described by the master equation

$$
\begin{aligned}
\dot{\rho}= & -\frac{i}{\hbar}\left[H+i \hbar \eta\left(\hat{a}^{\dagger} e^{-i \omega_{l} t}-\hat{a} e^{i \omega_{l} t}\right), \rho\right] \\
& +\kappa \mathcal{D}[\hat{a}] \rho+\sum_{i=1, \mu=A, B}^{i=N} \gamma_{i \mu} \mathcal{D}\left[\sigma_{i \mu}^{-}\right] \rho .
\end{aligned}
$$

Here, $\kappa$ is the decay rate of the cavity, and $\gamma_{i A}$ and $\gamma_{i B}$ are the decay rates of the qubits $A$ and $B$ at the $i$ th unit cell, respectively. The dissipation superoperator is defined as $\mathcal{D}[\hat{O}] \rho=\hat{O} \rho \hat{O}^{\dagger}-\frac{1}{2}\left\{\hat{O}^{\dagger} \hat{O}, \rho\right\}$. As shown in Fig. 1(d), the topological phase transition can be observed from the reflection of the probe field with special couplings between qubits and the cavity, which can be realized via controllable couplers [64-66]. The reflection spectrum is obtained by solving the master equation in Eq. (2) with $H$ given in Eq. (1). Topological phases have recently been demonstrated in superconducting qubit circuits [50,67-74]. However, the operations on topological states have not been implemented. Below we show that the topological bandgap is helpful for manipulations of edge states.

Vacuum Rabi splitting between the cavity and edge modes. We consider the single-excitation subspace consisting of $\left|\mathcal{A}_{i}\right\rangle=\sigma_{i A}^{+}|G\rangle$ and $\left|\mathcal{B}_{i}\right\rangle=\sigma_{i B}^{+}|G\rangle$ with $|G\rangle$ being the ground state of the qubit array. We rewrite the states $\left|\mathcal{A}_{i}\right\rangle$ and $\left|\mathcal{B}_{i}\right\rangle$ via eigenstates $\left|\Psi_{j}\right\rangle$ in the single-excitation subspace of the qubit array [47], i.e., $\left|\mathcal{A}_{i}\right\rangle=\sum_{j=1}^{2 N} \xi_{2 i-1, j}\left|\Psi_{j}\right\rangle$ and $\left|\mathcal{B}_{i}\right\rangle=$ $\sum_{j=1}^{2 N} \xi_{2 i, j}\left|\Psi_{j}\right\rangle$. Here, $j=1, \ldots, 2 N$ is the label of the $j$ th eigenstate from the lowest to highest energies. Then, the Hamiltonian in Eq. (1) can be rewritten as [64]

$$
\tilde{H} / \hbar=\sum_{j=1}^{2 N} \omega_{j} \Psi_{j}^{+} \Psi_{j}^{-}+\omega_{c} \hat{a}^{\dagger} \hat{a}+\sum_{j=1}^{2 N}\left(\tilde{\xi}_{j} \Psi_{j}^{+} \hat{a}+\text { H.c. }\right),
$$

with $\Psi_{j}^{+}=\left|\Psi_{j}\right\rangle\langle G|$, and the eigenfrequency $\omega_{j}$ corresponding to the eigenstate $\left|\Psi_{j}\right\rangle$. For homogeneous cavity-qubit couplings, i.e., $g_{i \mu} \equiv g_{0}$, the coupling strength between the cavity and the $j$ th eigenmode is $\tilde{\xi}_{j}=\xi_{j} g_{0}$ with coupling coefficient $\xi_{j}=\sum_{i}\left(\xi_{2 i-1, j}+\xi_{2 i, j}\right)$. The analytical expressions for $\xi_{j}$ can be found in Ref. [64]. Hereafter, we call $\Psi_{j}^{+}$bulk or edge modes when $\left|\Psi_{j}\right\rangle$ are bulk or edge eigenstates. In Fig. 2(a), we show $\left|\xi_{j}\right|$ for the qubit array size $2 N=36$. The bulk modes have different couplings to the cavity because of their parities of wave functions. The odd-parity bulk states, i.e., $\xi_{2 i-1, j}=-\xi_{2 N+2-2 i, j}$ and $\xi_{2 i, j}=-\xi_{2 N+1-2 i, j}$, have zero coupling to the cavity. However, the even-parity bulk states, i.e., $\xi_{2 i-1, j}=\xi_{2 N+2-2 i, j}$ and $\xi_{2 i, j}=\xi_{2 N+1-2 i, j}$, are coupled to the cavity [64]. Two edge states have equal coupling strengths to the cavity, i.e., $\xi_{18}=\xi_{19}$.

In Fig. 2(b), we show the energy splitting produced by the cavity-qubit couplings. We assume that the qubit frequency is $\omega_{0}=2 \pi \times 6 \mathrm{GHz}$. The anticrossing near the driving frequency $\omega_{l}=2 \pi \times 6 \mathrm{GHz}$ represents the Rabi splitting due to the resonant interaction between the cavity and edge modes. We also study the disorder effect and find that the Rabi 


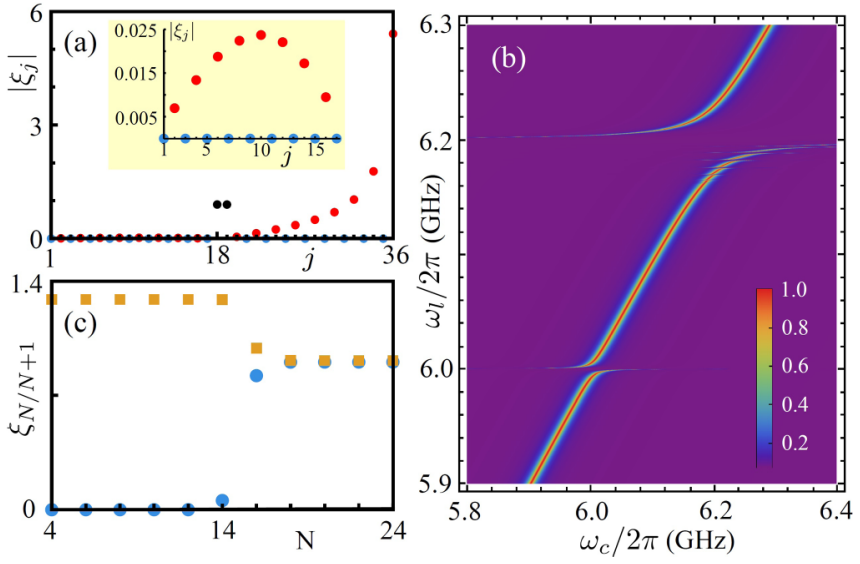

FIG. 2. (a) The coupling strength between the cavity and eigenmodes of the SSH qubit array with $N=18$ unit cells (i.e., 36 qubits) for homogeneous cavity-qubit couplings. The number $j$ is the index of eigenmodes, and the middle two points (black dots) with $j=18,19$ are edge states. The bulk states with odd (blue dots) and even (red dots) numbers have zero and nonzero couplings to the cavity, respectively. The inset represents the coupling strengths for $j<18$. (b) Vacuum Rabi splitting between edge states and the cavity for $N=18$ unit cells. The lower anticrossing shows the coupling between the cavity and the edge states. The upper one is the coupling of the bulk states to the cavity. (c) The coupling strengths $\xi_{N}$ and $\xi_{N+1}$, denoted by $\xi_{N / N+1}$, between two edge modes and the cavity with different numbers $N$ of unit cells. Here we consider $\varphi=\pi / 5$. Other parameters are the same as in Fig. 1(d).

splitting of edge states is robust to disorder [64]. If the frequency of the cavity is at resonance for the transitions from the ground to bulk states with high energies, a large anticrossing, as shown in upper part of Fig. 2(b), is produced around $\omega_{l}=2 \pi \times 6.2 \mathrm{GHz}$. The topological bandgap of the SSH Hamiltonian protects the Rabi splitting of edge states. In Fig. 2(c), $\xi_{N}$ and $\xi_{N+1}$, i.e., the coupling coefficients between the cavity and edge modes, are plotted versus the unit cell number $N$. When the qubit array is small, e.g., $N \leqslant 14$, the edge states overlap with each other and form hybridized edge states with odd and even parities. The edge state with odd parity decouples from the cavity. With the increase of the unit cell number, two edge states are far separated from each other. The localized edge states lose parity, thus they have the same coupling strength to the cavity.

We study the relation between the coupling coefficient $\xi_{N}$ $\left(\xi_{N+1}\right)$ and $\varphi$ in Fig. 3. For example, when the qubit array has $N=6$ unit cells, the coupling strengths are described by the black-solid and blue-dashed curves. When $\varphi$ is small, the edge states are unhybridized and have the same couplings to the cavity. However, the increase of $\varphi$ leads to hybridized edge states with even and odd parities. We find that the hybridized regime becomes smaller with the increase of the system size, e.g., $N=18$ (green-solid and blue-dash-dotted curves) and $N=78$ (red-solid and orange-dotted curves) as we show here. We also find that in the topological phase (i.e., $0 \leqslant \varphi<\pi / 2$ ), the hybridized edge state with even parity has the coupling strength $\tilde{\xi}_{+}=\sqrt{2 \cos \varphi} g_{0}$, which is independent of system size [64]. The couplings for separated edge states

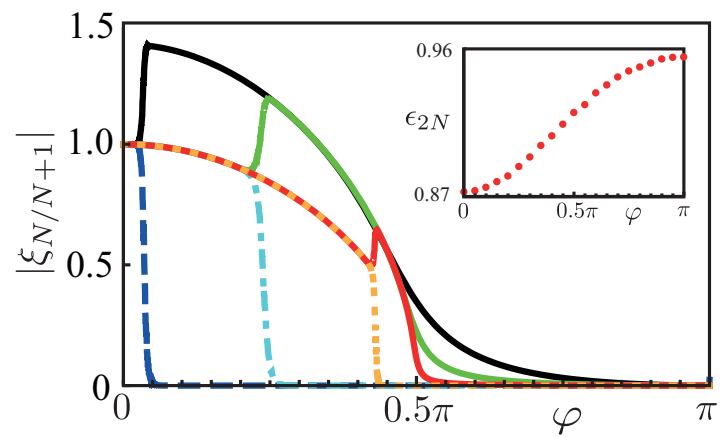

FIG. 3. The couplings between the cavity and edge states (blacksolid and blue-dashed curves) for the qubit array with $N=6$ unit cells. We also present the coupling strengths for $N=18(N=78)$ by the green-solid and blue-dash-dotted (red-solid and orange-dotted) curves. The inset shows the coefficient $\epsilon_{2 N}$ of the collective coupling $\tilde{\xi}_{j}=\epsilon_{j} \sqrt{2 N} g_{0}$ with $j=2 N$.

are $\tilde{\xi}_{L}=\tilde{\xi}_{R}=\sqrt{\cos \varphi} g_{0}$. Because the coupling strength of $2 N$ noninteracting qubits to the cavity is $\sqrt{2 N} g_{0}$ [75], we here assume that the coupling strength of $2 N$ interacting qubits to the cavity is $\tilde{\xi}_{j}=\epsilon_{j} \sqrt{2 N} g_{0}$ with the rescaling factor $\epsilon_{j}$ given in Eq. (S29) of the Supplemental Material [64]. As shown in the inset of Fig. 3, the rescaling factor $\epsilon_{2 N}$ is tuned by $\varphi$.

Topological-bandgap-protected coupling between two edge modes. When the cavity is far detuned from qubits, i.e., $g_{0} \ll$ $\Delta_{0}$ (let $\Delta_{0}=\omega_{0}-\omega_{c}$ ), virtual-photon-mediated interactions among qubits $g_{0}^{2} / \Delta_{0}$ can be obtained [39,61], as shown in Fig. 1(e). In terms of eigenmodes of the qubit array, the effective coupling strengths between $j$ th and $k$ th eigenmodes are

$$
J_{j k}=\frac{\tilde{\xi}_{j} \tilde{\xi}_{k}}{2}\left(\frac{1}{\Delta_{j}}+\frac{1}{\Delta_{k}}\right), \quad j, k \in[1, \ldots, 2 N],
$$

with $\Delta_{j / k}=\omega_{j / k}-\omega_{c}$, which depends on system size $2 N$ for the coupling between bulk modes, or between bulk and edge modes, due to the size-dependent cavity-bulk coupling. However, the edge-mode coupling is independent of the size, i.e.,

$$
J=\cos \varphi \frac{g_{0}^{2}}{\Delta_{0}},
$$

which is protected by the topological bandgap, as schematically shown in Fig. 4(a). We note that the energy splitting induced by hybridization of edge states is assumed to be negligibly small when Eq. (5) is derived. In Figs. 4(b) and 4(c), we show the excitation dynamics of the left-edge qubit (qubit $A$ in the first unit cell is excited initially) in the topological phase with $\varphi=0.1 \pi$ and $0.3 \pi$, respectively. Figures 4(b) and 4(c) clearly show the population exchange between two edge states produced by the edge-mode coupling. In fact, the finite topological bandgap makes the effective couplings between edge modes different from Eq. (5) [64]. In Figs. 4(d) and $4(\mathrm{e})$ with $\varphi=0.5 \pi$ and $0.9 \pi$, the excitation propagates through the array and is bounded by the boundaries. In the nontopological phase, excitation propagates along the qubit array with low velocity [see Fig. 4(e)], which is yielded by the smooth energy bands with large gap. 


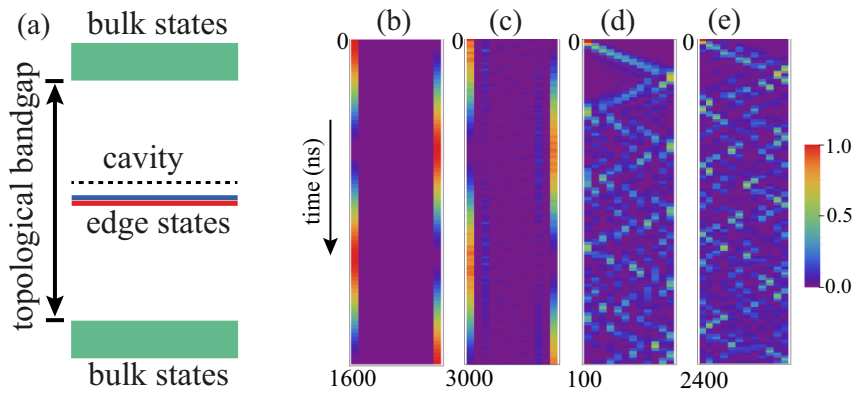

FIG. 4. (a) Edge-state coupling protected by the topological bandgap. (b)-(e) Excitation dynamics in the cavity-coupled SSH qubit array for $\varphi=0.1 \pi, 0.3 \pi, 0.5 \pi$, and $0.9 \pi$. Horizontal and vertical axes denote qubits and time, respectively. The virtual-photonmediated interactions among qubits are assumed to be $0.1 g_{0}$. The number of unit cells is $N=6$, and other parameters are the same as in Fig. 1(d).

Quantum interference induced by topological state coupling. As schematically shown in Fig. 5(a), we further consider that the left-edge qubit $A_{1}$ is coupled to a waveguide, in which a probe field passes through. The left-edge qubit mainly contributes to the left edge state. Then the left edge state can be driven by fields passing through the waveguide. The single-photon transmission amplitude can be given as [64]

$$
t=\frac{\left(i \Delta_{p}-\frac{\gamma_{L}}{2}\right)\left(i \Delta_{p}-\frac{\gamma_{R}}{2}\right)+J^{2}}{\left(i \Delta_{p}-\frac{\gamma_{L}+\Gamma_{L}}{2}\right)\left(i \Delta_{p}-\frac{\gamma_{R}}{2}\right)+J^{2}},
$$

and the susceptibility $\chi=-i(t-1) / t$ is

$$
\chi=\frac{\Gamma_{L}\left(\Delta_{p}+i \frac{\gamma_{R}}{2}\right)}{2 J^{2}-2\left(\Delta_{p}+i \frac{\gamma_{L}}{2}\right)\left(\Delta_{p}+i \frac{\gamma_{R}}{2}\right)},
$$

where $\Delta_{p}$ is the detuning between the probe field and the left edge state. As schematically shown in Fig. 5(b), the parameters $\gamma_{L}$ and $\gamma_{R}$ are the decay rates for left and right edge states, and $\Gamma_{L}$ comes from the coupling between the left edge qubit and the waveguide.

The transmission of the probe field as a function of the detuning $\Delta_{p}$ is shown in Fig. 5(c) with $J=0$ and $0.035 \Gamma_{L}$. When there is no coupling between edge states, the transmission vanishes at the resonance. However, when there is a coupling between two edge states, a transparency window for the probe field appears. This can be further confirmed by the susceptibility, which is plotted as a function of the detuning $\Delta_{p}$ in Fig. $5(\mathrm{~d})$ in the parameter regime $J \ll \Gamma_{L}$. This transparency window, in which the distance between two peaks is less than $2 J$, is from the quantum interference as shown in Fig. 5(e), which is similar to electromagnetically induced transparency [76]. However, in the parameter regime $J>\Gamma_{L}$, the transparency window, in which the distance between two peaks equals to $2 J$, is from the strong-couplinginduced energy splitting, which is similar to Autler-Townes splitting [77].

Discussions and conclusions. In summary, we study cavity control of topological edge states in SSH qubit arrays. We show that the couplings between cavity and edge modes are protected by the topological bandgap, and topological phase
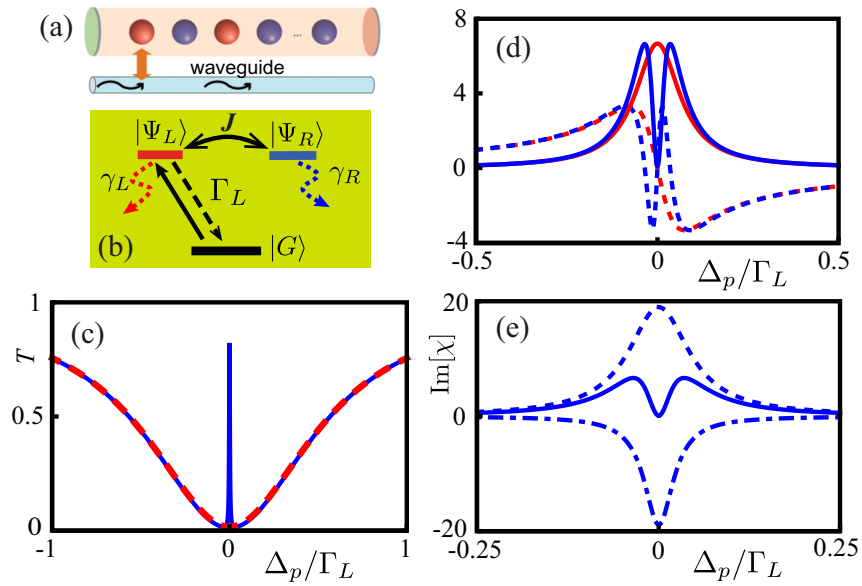

FIG. 5. (a) Coupling between a waveguide and the left-edge qubit in the array. (b) Superatom model of (a). Here $\gamma_{L}$ and $\gamma_{R}$ are respective decays of left and right edge states, and $\Gamma_{L}$ is the waveguide induced decay rate of the left edge state. (c) Transmission of probe light for edge-state coupling $J=0\left(J=0.035 \Gamma_{L}\right)$ is represented by the red-dashed (blue-solid) curve. (d) Real (solid) and imaginary (dashed) parts of the susceptibility. In both (c) and (d), the red and blue curves are for $J=0$ and $J=0.035 \Gamma_{L}$, respectively. (e) The imaginary part of susceptibility can be decomposed into two Lorentzian peaks. In these three figures, we consider $\gamma_{L}=0.15 \Gamma_{L}$, $\gamma_{R}=5 \times 10^{-4} \Gamma_{L}$.

transitions can be probed via the reflection spectrum of the probe field through the cavity. Due to the bandgap, Rabi splitting of edge modes can be observed. When the cavity is largely detuned from the edge modes, long-range coupling between two edge states can be realized. It results in quantum interference for emissions from two edge states when a qubit at the edge of the array is coupled to a waveguide. Meanwhile, we find that topological properties can also be detected by the cavity even for a small system, where the edge states are hybridized.

We also discuss experimental feasibility via a superconducting qubit array coupled to a microwave transmission line resonator [44]. The tunable couplings between qubits [62] or between qubits and the resonator [65] make our proposal more experimentally accessible. We also analyze the effects of disorder and decay on the results. We find that both the Rabi splitting and excitation dynamics produced by edge-state coupling are robust to the disorder [64]. We also find that with current coherence time $20-40 \mu$ s in 1D arrays [41,42], the Rabi splitting, excitation dynamics, and quantum interference induced by the edge-state coupling should be observed. We mention that our approach can also be applied to other topological quantum systems. Our study here might have potential applications in quantum information and quantum optics.

Acknowledgments. This work is supported by the National Basic Research Program (973) of China under Grant No. 2017YFA0304304 and the Key-Area R\&D Program of GuangDong Province under Grant No. 2018B030326001. W.N. acknowledges the Tsinghua University Postdoctoral Support Program. 
[1] A. Bansil, H. Lin, and T. Das, Colloquium: Topological band theory, Rev. Mod. Phys. 88, 021004 (2016).

[2] D. J. Thouless, M. Kohmoto, M. P. Nightingale, and M. den Nijs, Quantized Hall Conductance in a Two-Dimensional Periodic Potential, Phys. Rev. Lett. 49, 405 (1982).

[3] J. Zak, Berry's Phase for Energy Bands in Solids, Phys. Rev. Lett. 62, 2747 (1989).

[4] D. Xiao, M.-C. Chang, and Q. Niu, Berry phase effects on electronic properties, Rev. Mod. Phys. 82, 1959 (2010).

[5] K. T. Law, P. A. Lee, and T. K. Ng, Majorana Fermion Induced Resonant Andreev Reflection, Phys. Rev. Lett. 103, 237001 (2009).

[6] L. Fu, Electron Teleportation via Majorana Bound States in a Mesoscopic Superconductor, Phys. Rev. Lett. 104, 056402 (2010).

[7] T. Karzig, C. Knapp, R. M. Lutchyn, P. Bonderson, M. B. Hastings, C. Nayak, J. Alicea, K. Flensberg, S. Plugge, Y. Oreg, C. M. Marcus, and M. H. Freedman, Scalable designs for quasiparticle-poisoning-protected topological quantum computation with Majorana zero modes, Phys. Rev. B 95, 235305 (2017).

[8] T. E. O’Brien, P. Rożek, and A. R. Akhmerov, Majorana-Based Fermionic Quantum Computation, Phys. Rev. Lett. 120, 220504 (2018).

[9] Y. Li, Fault-tolerant fermionic quantum computation based on color code, Phys. Rev. A 98, 012336 (2018).

[10] V. Mourik, K. Zuo, S. M. Frolov, S. R. Plissard, E. P. A. M. Bakkers, and L. P. Kouwenhoven, Signatures of Majorana fermions in hybrid superconductor-semiconductor nanowire devices, Science 336, 1003 (2012).

[11] M. T. Deng, C. L. Yu, G. Y. Huang, M. Larsson, P. Caroff, and $\mathrm{H}$. Q. Xu, Anomalous zero-bias conductance peak in a $\mathrm{Nb}-\mathrm{InSb}$ nanowire-Nb hybrid device, Nano Lett. 12, 6414 (2012).

[12] S. M. Albrecht, A. P. Higginbotham, M. Madsen, F. Kuemmeth, T. S. Jespersen, J. Nygård, P. Krogstrup, and C. M. Marcus, Exponential protection of zero modes in Majorana islands, Nature (London) 531, 206 (2016).

[13] H. Zhang, C.-X. Liu, S. Gazibegovic, D. Xu, J. A. Logan, G. Wang, N. van Loo, J. D. S. Bommer, M. W. A. de Moor, D. Car, R. L. M. Op het Veld, P. J. van Veldhoven, S. Koelling, M. A. Verheijen, M. Pendharkar, D. J. Pennachio, B. Shojaei, J. S. Lee, C. J. Palmstrøm, E. P. A. M. Bakkers, S. D. Sarma, and L. P. Kouwenhoven, Quantized Majorana conductance, Nature (London) 556, 74 (2018).

[14] S. Nadj-Perge, I. K. Drozdov, J. Li, H. Chen, S. Jeon, J. Seo, A. H. MacDonald, B. A. Bernevig, and A. Yazdani, Observation of Majorana fermions in ferromagnetic atomic chains on a superconductor, Science 346, 602 (2014).

[15] D. Wang, L. Kong, P. Fan, H. Chen, S. Zhu, W. Liu, L. Cao, Y. Sun, S. Du, J. Schneeloch, R. Zhong, G. Gu, L. Fu, H. Ding, and H.-J. Gao, Evidence for Majorana bound states in an iron-based superconductor, Science 362, 333 (2018).

[16] Y. Xia, D. Qian, D. Hsieh, L. Wray, A. Pal, H. Lin, A. Bansil, D. Grauer, Y. S. Hor, R. J. Cava, and M. Z. Hasan, Observation of a large-gap topological-insulator class with a single Dirac cone on the surface, Nat. Phys. 5, 398 (2009).

[17] J. Hu, J. Alicea, R. Wu, and M. Franz, Giant Topological Insulator Gap in Graphene with $5 d$ Adatoms, Phys. Rev. Lett. 109, 266801 (2012).
[18] Y. Xu, B. Yan, H.-J. Zhang, J. Wang, G. Xu, P. Tang, W. Duan, and S.-C. Zhang, Large-Gap Quantum Spin Hall Insulators in Tin Films, Phys. Rev. Lett. 111, 136804 (2013).

[19] S. Haroche and J.-M. Raimond, Exploring the Quantum: Atoms, Cavities, and Photons (Oxford University Press, 2006).

[20] H. Ritsch, P. Domokos, F. Brennecke, and T. Esslinger, Cold atoms in cavity-generated dynamical optical potentials, Rev. Mod. Phys. 85, 553 (2013).

[21] A. Reiserer and G. Rempe, Cavity-based quantum networks with single atoms and optical photons, Rev. Mod. Phys. 87, 1379 (2015).

[22] A. Blais, R.-S. Huang, A. Wallraff, S. M. Girvin, and R. J. Schoelkopf, Cavity quantum electrodynamics for superconducting electrical circuits: An architecture for quantum computation, Phys. Rev. A 69, 062320 (2004).

[23] A. Wallraff, D. I. Schuster, A. Blais, L. Frunzio, R.-S. Huang, J. Majer, S. Kumar, S. M. Girvin, and R. J. Schoelkopf, Strong coupling of a single photon to a superconducting qubit using circuit quantum electrodynamics, Nature (London) 431, 162 (2004).

[24] X. Gu, A. F. Kockum, A. Miranowicz, Y.-X. Liu, and F. Nori, Microwave photonics with superconducting quantum circuits, Phys. Rep. 718-719, 1 (2017).

[25] M. H. Devoret and R. J. Schoelkopf, Superconducting circuits for quantum information: An outlook, Science 339, 1169 (2013).

[26] Y.-D. Wang, F. Xue, Z. Song, and C.-P. Sun, Detection mechanism for quantum phase transition in superconducting qubit array, Phys. Rev. B 76, 174519 (2007).

[27] Z.-Y. Xue, S.-L. Zhu, J. Q. You, and Z. D. Wang, Implementing topological quantum manipulation with superconducting circuits, Phys. Rev. A 79, 040303(R) (2009).

[28] L. Tian, Circuit QED and Sudden Phase Switching in a Superconducting Qubit Array, Phys. Rev. Lett. 105, 167001 (2010).

[29] O. Viehmann, J. von Delft, and F. Marquardt, Observing the Nonequilibrium Dynamics of the Quantum Transverse-Field Ising Chain in Circuit QED, Phys. Rev. Lett. 110, 030601 (2013).

[30] D. Marcos, P. Rabl, E. Rico, and P. Zoller, Superconducting Circuits for Quantum Simulation of Dynamical Gauge Fields, Phys. Rev. Lett. 111, 110504 (2013).

[31] J. Q. You, Z. D. Wang, W. X. Zhang, and F. Nori, Encoding a qubit with Majorana modes in superconducting circuits, Sci. Rep. 4, 5535 (2014).

[32] A. Kurcz, A. Bermudez, and J. J. García-Ripoll, Hybrid Quantum Magnetism in Circuit QED: From Spin-Photon Waves to Many-Body Spectroscopy, Phys. Rev. Lett. 112, 180405 (2014).

[33] Q.-M. Chen, Y.-X. Liu, L. Sun, and R.-B. Wu, Tuning the coupling between superconducting resonators with collective qubits, Phys. Rev. A 98, 042328 (2018).

[34] P. Macha, G. Oelsner, J.-M. Reiner, M. Marthaler, S. André, G. Schön, U. Hübner, H.-G. Meyer, E. Il'ichev, and A. V. Ustinov, Implementation of a quantum metamaterial using superconducting qubits, Nat. Commun. 5, 5146 (2014).

[35] R. Barends, A. Shabani, L. Lamata, J. Kelly, A. Mezzacapo, U. L. Heras, R. Babbush, A. G. Fowler, B. Campbell, Y. Chen, Z. Chen, B. Chiaro, A. Dunsworth, E. Jeffrey, E. Lucero, A. Megrant, J. Y. Mutus, M. Neeley, C. Neill, P. J. J. O’Malley, C. Quintana, P. Roushan, D. Sank, A. Vainsencher, J. Wenner, T. C. White, E. Solano, H. Neven, and J. M. Martinis, Digitized 
adiabatic quantum computing with a superconducting circuit, Nature (London) 534, 222 (2016).

[36] K. Kakuyanagi, Y. Matsuzaki, C. Déprez, H. Toida, K. Semba, H. Yamaguchi, W. J. Munro, and S. Saito, Observation of Collective Coupling between an Engineered Ensemble of Macroscopic Artificial Atoms and a Superconducting Resonator, Phys. Rev. Lett. 117, 210503 (2016).

[37] M. Fitzpatrick, N. M. Sundaresan, A. C. Y. Li, J. Koch, and A. A. Houck, Observation of a Dissipative Phase Transition in a One-Dimensional Circuit QED Lattice, Phys. Rev. X 7, 011016 (2017).

[38] P. Roushan, C. Neill, J. Tangpanitanon, V. M. Bastidas, A. Megrant, R. Barends, Y. Chen, Z. Chen, B. Chiaro, A. Dunsworth, A. Fowler, B. Foxen, M. Giustina, E. Jeffrey, J. Kelly, E. Lucero, J. Mutus, M. Neeley, C. Quintana, D. Sank, A. Vainsencher, J. Wenner, T. White, H. Neven, D. G. Angelakis, and J. Martinis, Spectroscopic signatures of localization with interacting photons in superconducting qubits, Science $\mathbf{3 5 8}$, 1175 (2017).

[39] K. Xu, J.-J. Chen, Y. Zeng, Y.-R. Zhang, C. Song, W. Liu, Q. Guo, P. Zhang, D. Xu, H. Deng, K. Huang, H. Wang, X. Zhu, D. Zheng, and H. Fan, Emulating Many-Body Localization with a Superconducting Quantum Processor, Phys. Rev. Lett. 120, 050507 (2018).

[40] M. Mirhosseini, E. Kim, X. Zhang, A. Sipahigil, P. B. Dieterle, A. J. Keller, A. Asenjo-Garcia, D. E. Chang, and O. Painter, Cavity quantum electrodynamics with atom-like mirrors, Nature (London) 569, 692 (2019).

[41] R. Ma, B. Saxberg, C. Owens, N. Leung, Y. Lu, J. Simon, and D. I. Schuster, A dissipatively stabilized Mott insulator of photons, Nature (London) 566, 51 (2019).

[42] Z. Yan, Y.-R. Zhang, M. Gong, Y. Wu, Y. Zheng, S. Li, C. Wang, F. Liang, J. Li, Y. Xu, C. Guo, L. Sun, C.-Z. Peng, K. Xia, H. Deng, H. Rong, J. Q. You, F. Nori, H. Fan, X. Zhu, and J.-W. Pan, Strongly correlated quantum walks with a 12-qubit superconducting processor, Science 364, 753 (2019).

[43] J. Tangpanitanon, V. M. Bastidas, S. Al-Assam, P. Roushan, D. Jaksch, and D. G. Angelakis, Topological Pumping of Photons in Nonlinear Resonator Arrays, Phys. Rev. Lett. 117, 213603 (2016).

[44] X. Gu, S. Chen, and Y.-X. Liu, Topological edge states and pumping in a chain of coupled superconducting qubits, arXiv:1711.06829.

[45] F. Mei, G. Chen, L. Tian, S.-L. Zhu, and S. Jia, Robust quantum state transfer via topological edge states in superconducting qubit chains, Phys. Rev. A 98, 012331 (2018).

[46] F. Mei, G. Chen, L. Tian, S.-L. Zhu, and S. Jia, Topologydependent quantum dynamics and entanglement-dependent topological pumping in superconducting qubit chains, Phys. Rev. A 98, 032323 (2018).

[47] W. Nie, Z. H. Peng, F. Nori, and Y.-X. Liu, Topological Quantum Coherence in a Symmetry-Protected Superatom, Phys. Rev. Lett. 124, 023603 (2020).

[48] W. P. Su, J. R. Schrieffer, and A. J. Heeger, Solitons in Polyacetylene, Phys. Rev. Lett. 42, 1698 (1979).

[49] M. Atala, M. Aidelsburger, J. T. Barreiro, D. Abanin, T. Kitagawa, E. Demler, and I. Bloch, Direct measurement of the Zak phase in topological Bloch bands, Nat. Phys. 9, 795 (2013).

[50] W. Cai, J. Han, F. Mei, Y. Xu, Y. Ma, X. Li, H. Wang, Y. P. Song, Z.-Y. Xue, Z.-Q. Yin, S. Jia, and L. Sun, Observation of Topological Magnon Insulator States in a Superconducting Circuit, Phys. Rev. Lett. 123, 080501 (2019).

[51] S. de Léséleuc, V. Lienhard, P. Scholl, D. Barredo, S. Weber, N. Lang, H. P. Büchler, T. Lahaye, and A. Browaeys, Observation of a symmetry-protected topological phase of interacting bosons with Rydberg atoms, Science 365, 775 (2019).

[52] E. Prada, P. San-Jose, and R. Aguado, Transport spectroscopy of $N S$ nanowire junctions with Majorana fermions, Phys. Rev. B 86, 180503(R) (2012).

[53] J. Liu, A. C. Potter, K. T. Law, and P. A. Lee, Zero-Bias Peaks in the Tunneling Conductance of Spin-Orbit-Coupled Superconducting Wires with and without Majorana End-States, Phys. Rev. Lett. 109, 267002 (2012).

[54] D. Rainis, L. Trifunovic, J. Klinovaja, and D. Loss, Towards a realistic transport modeling in a superconducting nanowire with Majorana fermions, Phys. Rev. B 87, 024515 (2013).

[55] S.-Q. Shen, Topological Insulators, 2nd ed. (Springer-Verlag, Berlin, 2017).

[56] A. Blais, A. Maassen van den Brink, and A. M. Zagoskin, Tunable Coupling of Superconducting Qubits, Phys. Rev. Lett. 90, 127901 (2003)

[57] Y.-X. Liu, L. F. Wei, J. S. Tsai, and F. Nori, Controllable Coupling between Flux Qubits, Phys. Rev. Lett. 96, 067003 (2006).

[58] M. Grajcar, Y.-X. Liu, F. Nori, and A. M. Zagoskin, Switchable resonant coupling of flux qubits, Phys. Rev. B 74, 172505 (2006).

[59] S. H. W. van der Ploeg, A. Izmalkov, A. M. van den Brink, U. Hübner, M. Grajcar, E. Il'ichev, H.-G. Meyer, and A. M. Zagoskin, Controllable Coupling of Superconducting Flux Qubits, Phys. Rev. Lett. 98, 057004 (2007).

[60] A. O. Niskanen, K. Harrabi, F. Yoshihara, Y. Nakamura, S. Lloyd, and J. S. Tsai, Quantum coherent tunable coupling of superconducting qubits, Science 316, 723 (2007).

[61] J. Majer, J. M. Chow, J. M. Gambetta, J. Koch, B. R. Johnson, J. A. Schreier, L. Frunzio, D. I. Schuster, A. A. Houck, A. Wallraff, A. Blais, M. H. Devoret, S. M. Girvin, and R. J. Schoelkopf, Coupling superconducting qubits via a cavity bus, Nature (London) 449, 443 (2007).

[62] Y. Chen, C. Neill, P. Roushan, N. Leung, M. Fang, R. Barends, J. Kelly, B. Campbell, Z. Chen, B. Chiaro, A. Dunsworth, E. Jeffrey, A. Megrant, J. Y. Mutus, P. J. J. O'Malley, C. M. Quintana, D. Sank, A. Vainsencher, J. Wenner, T. C. White, M. R. Geller, A. N. Cleland, and J. M. Martinis, Qubit Architecture with High Coherence and Fast Tunable Coupling, Phys. Rev. Lett. 113, 220502 (2014).

[63] M. R. Geller, E. Donate, Y. Chen, M. T. Fang, N. Leung, C. Neill, P. Roushan, and J. M. Martinis, Tunable coupler for superconducting Xmon qubits: Perturbative nonlinear model, Phys. Rev. A 92, 012320 (2015).

[64] See Supplemental Material at http://link.aps.org/supplemental/ 10.1103/PhysRevResearch.2.012076 for additional details about tunable couplings in the SSH qubit array, the sign of the cavity-qubit coupling, analytical expressions for the couplings between eigenmodes and cavity, cavity spectroscopy of SSH qubit array, quantum dynamics protected by topological bandgap, and quantum interference produced by edge-state coupling.

[65] Y. P. Zhong, H.-S. Chang, K. J. Satzinger, M.-H. Chou, A. Bienfait, C. R. Conner, É. Dumur, J. Grebel, G. A. Peairs, 
R. G. Povey, D. I. Schuster, and A. N. Cleland, Violating Bell's inequality with remotely connected superconducting qubits, Nat. Phys. 15, 741 (2019).

[66] H.-K. Li, K.-M. Li, H. Dong, Q.-J. Guo, W.-X. Liu, Z. Wang, H.-H. Wang, and D.-N. Zheng, Tunable coupling between Xmon qubit and coplanar waveguide resonator, Chin. Phys. B 28, 080305 (2019).

[67] M. D. Schroer, M. H. Kolodrubetz, W. F. Kindel, M. Sandberg, J. Gao, M. R. Vissers, D. P. Pappas, A. Polkovnikov, and K. W. Lehnert, Measuring a Topological Transition in an Artificial Spin-1/2 System, Phys. Rev. Lett. 113, 050402 (2014).

[68] P. Roushan, C. Neill, Y. Chen, M. Kolodrubetz, C. Quintana, N. Leung, M. Fang, R. Barends, B. Campbell, Z. Chen, B. Chiaro, A. Dunsworth, E. Jeffrey, J. Kelly, A. Megrant, J. Mutus, P. J. J. O’Malley, D. Sank, A. Vainsencher, J. Wenner, T. White, A. Polkovnikov, A. N. Cleland, and J. M. Martinis, Observation of topological transitions in interacting quantum circuits, Nature (London) 515, 241 (2014).

[69] E. Flurin, V. V. Ramasesh, S. Hacohen-Gourgy, L. S. Martin, N. Y. Yao, and I. Siddiqi, Observing Topological Invariants Using Quantum Walks in Superconducting Circuits, Phys. Rev. X 7, 031023 (2017).

[70] T. Wang, Z. Zhang, L. Xiang, Z. Gong, J. Wu, and Y. Yin, Simulating a topological transition in a superconducting phase qubit by fast adiabatic trajectories, Sci. China Phys. Mech. Astron. 61, 047411 (2018).

[71] X. Tan, D.-W. Zhang, Q. Liu, G. Xue, H.-F. Yu, Y.-Q. Zhu, H. Yan, S.-L. Zhu, and Y. Yu, Topological Maxwell Metal Bands in a Superconducting Qutrit, Phys. Rev. Lett. 120, 130503 (2018).
[72] C. Song, D. Xu, P. Zhang, J. Wang, Q. Guo, W. Liu, K. Xu, H. Deng, K. Huang, D. Zheng, S.-B. Zheng, H. Wang, X. Zhu, C.-Y. Lu, and J.-W. Pan, Demonstration of Topological Robustness of Anyonic Braiding Statistics with a Superconducting Quantum Circuit, Phys. Rev. Lett. 121, 030502 (2018).

[73] A. D. King, J. Carrasquilla, J. Raymond, I. Ozfidan, E. Andriyash, A. Berkley, M. Reis, T. Lanting, R. Harris, F. Altomare, K. Boothby, P. I. Bunyk, C. Enderud, A. Fréchette, E. Hoskinson, N. Ladizinsky, T. Oh, G. Poulin-Lamarre, C. Rich, Y. Sato, A. Y. Smirnov, L. J. Swenson, M. H. Volkmann, J. Whittaker, J. Yao, E. Ladizinsky, M. W. Johnson, J. Hilton, and M. H. Amin, Observation of topological phenomena in a programmable lattice of 1,800 qubits, Nature (London) 560, 456 (2018).

[74] X. Tan, Y. X. Zhao, Q. Liu, G. Xue, H.-F. Yu, Z. D. Wang, and Y. $\mathrm{Yu}$, Simulation and Manipulation of Tunable Weyl-Semimetal Bands Using Superconducting Quantum Circuits, Phys. Rev. Lett. 122, 010501 (2019).

[75] M. Tavis and F. W. Cummings, Exact Solution for an $\mathrm{N}$ Molecule-Radiation-Field Hamiltonian, Phys. Rev. 170, 379 (1968).

[76] J. Long, H. S. Ku, X. Wu, X. Gu, R. E. Lake, M. Bal, Y.-X. Liu, and D. P. Pappas, Electromagnetically Induced Transparency in Circuit Quantum Electrodynamics with Nested Polariton States, Phys. Rev. Lett. 120, 083602 (2018).

[77] M. A. Sillanpää, J. Li, K. Cicak, F. Altomare, J. I. Park, R. W. Simmonds, G. S. Paraoanu, and P. J. Hakonen, Autler-Townes Effect in a Superconducting Three-Level System, Phys. Rev. Lett. 103, 193601 (2009). 